\title{
All-Optical Phase Regeneration of Multi-level Amplitude and Phase Shift Keyed Signals
}

\author{
G. Hesketh, P. Horak \\ 1. Optoelectronics Research Centre, University of Southampton, Southampton SO17 1BJ, UK
}

\begin{abstract}
All-optical phase regeneration of multi-level phase shift keyed signals in fiber optic communications systems reduces the impact of phase noise induced by, e.g., self/cross-phase modulation of amplitude varying symbols, and has been previously simulated and experimentally demonstrated [1-3]. Phase regeneration is achieved through a judicious coherent addition of phase harmonics, bearing integer multiples of the signal phase, to the signal via four-wave mixing in a highly nonlinear fiber (HNLF), such that a staircase is realised in the signal phase transfer function of the HNLF. Phase regeneration may allow for greater transmission capacity and optical regeneration has the potential to work faster and consume less power than electronic compensation.

In [1-2], the 2-term phase transfer function is excellent at squeezing phases to desired constellation points, yet it suffers notable phase-to-amplitude noise conversion rendering it less able to operate in multi-level amplitude and phase shift keying (APSK) formats. Here, we introduce a 3-term phase transfer function capable of phase regeneration of APSK formats without phase-to-amplitude noise conversion. We numerically solve a nonlinear Schrödinger equation to show four-wave mixing driven by three phase-locked pumps combining a signal and two phase harmonics in a HNLF; the phase harmonics and pumps are generated by a frequency comb and the four-wave mixing is nonlinear in pump powers but approximately linear in signal and phase harmonic powers thus accommodating multi-level amplitudes. By adjusting pump powers we tune weights $m_{1}$ and $m_{2}$ in the following signal transfer function:
\end{abstract}

$$
A_{\text {out }} e^{i \phi_{\text {out }}} \propto e^{i \phi_{\text {in }}}+m_{1} e^{-i(M-1) \phi_{\text {in }}}+m_{2} e^{i(M+1) \phi_{\text {in }}},
$$

where $A, \phi$ and $M$ are the signal amplitude, phase and integer phase quantization level respectively. The novel feature of this work is the inclusion of the third term: when tuned appropriately it reduces amplitude oscillations in the transfer function yet preserves the phase staircase. As an example, Fig 1 shows the phase regeneration of a 4-phase, 2-amplitude level APSK format. To simulate noise, the data is encoded on Gaussian pulses in a wavelength-division multiplexing system, propagated through five $100 \mathrm{~km}$ spans of a communications link at high peak power, frequency demuxed and then passed through the regenerative section twice to maximize phase squeezing; two tunings of the phase squeezing transfer function are shown for comparison. Using the $m_{1}=$ $0.5, m_{2}=0$ tuning [1-2], is inadequate here and the phase to amplitude noise conversion is seen to merge the two amplitude levels. The transfer function presented herein tuned with $m_{1}=0.14, m_{2}=-0.14$, shows significant improvement in this regard. Alternatively, if working only with phase shift keyed signals and a broad flat phase step is desirable then the tuning $m_{1}=0.64, m_{2}=0.27$ is more appropriate. We will discuss various system design optimisations, including how best to tune the transfer function in various scenarios, where best to place it in a link, and its effect on information capacity.
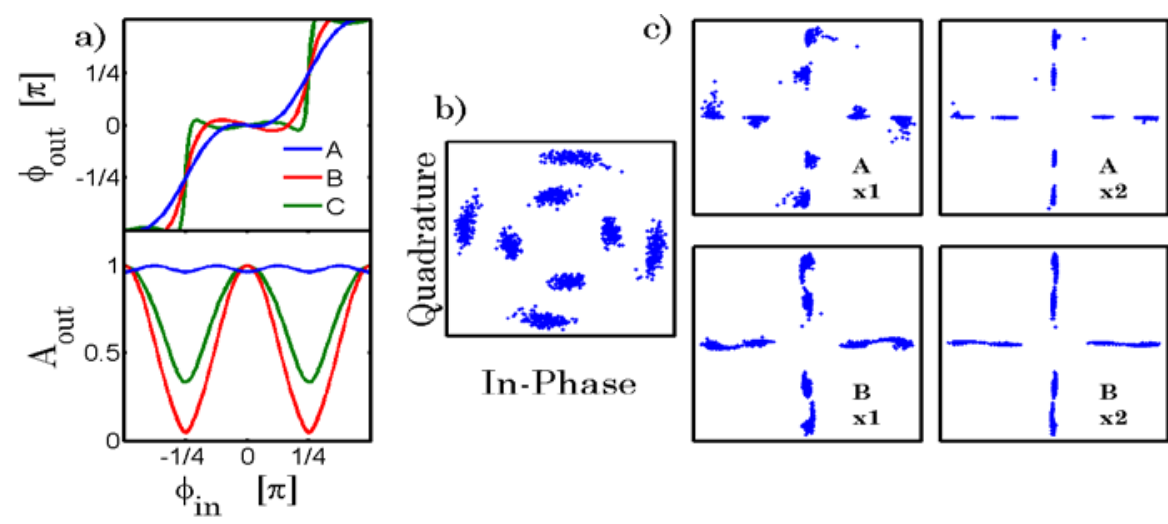

Fig. 1 a) Phase and amplitude signal transfer functions, A: flat amplitude, $m_{1}=0.14, m_{2}=-0.14$, B: tuning in $[1,2], m_{1}=0.5, m_{2}=0$, C: broad phase step, $m_{1}=0.64, m_{2}=0.27$ (C). b) Noisy simulated APSK constellation and c) the same distribution phase regenerated once (left) then again (right) using transfer functions A (top) and B (bottom). A maintains the distinct amplitude levels while B does not.

\section{References}

[1] J. Kakande et al., "Multilevel quantization of optical phase in a novel coherent parametric mixer architecture”, Nature, 5, 748 (2011).

[2] J. Kakande et al., "All-Optical Processing of Multilevel Phase Shift Keyed Signals", OFC (2012).

[3] J. Yang et al., "Optical Phase Regeneration of Multi-Level PSK Using Dual-Conjugate-Pump Degenerate Phase Sensitive

Amplification”, ECOC (2012) 